\title{
Relation between depression after stroke, antidepressant therapy, and functional recovery
}

\author{
G Gainotti, G Antonucci, C Marra, S Paolucci
}

\begin{abstract}
The aim was to evaluate the effects of poststroke depression and antidepressant therapy on the improvement of motor scores and disability, to verify if the negative effects of poststroke depression on functional recovery could be counterbalanced by taking antidepressant drugs.

Results obtained before, during, and after rehabilitation-on the Barthel index, Canadian neurological scale, and Rivermead mobility index-by 49 depressed patients with stroke, who had been treated $(n=25)$ or not treated $(n=24)$ according to the different therapeutic approaches of their physicians, were compared with results similarly obtained by 15 non-depressed patients with stroke. Analysis was by multivariate analysis of variance for repeated measures
\end{abstract}

There was a non-significant difference between the groups in their motor and functional scores, and a significant improvement on time. A significant interaction between group and time was seen. This interaction was particularly significant on the Rivermead mobility index, and was due to the fact that the recovery of non-treated depressed patients with stroke was less than the non-depressed and the depressed but treated patients with stroke. Furthermore, recovery from depression was significantly greater in treated than in non-treated depressed patients with stroke.

In conclusion, poststroke depression has negative effects on functional recovery, and a pharmacological treatment of depression can counterbalance this effect. (F Neurol Neurosurg Psychiatry 2001;71:258-261)

Keywords: poststroke depression; antidepressant drugs; functional recovery

Depression is a frequent and important problem of patients with stroke, as poststroke depression is present in at least $30 \%$ of survivors from stroke, ${ }^{1-3}$ and has a deleterious effect not only on the motivation, but also on the cognitive functions of these patients. ${ }^{4}$ It should be logical to expect that poststroke depression has a negative impact on functional recovery but its effects on the outcome of rehabilitation remain controversial. Some authors $5^{5} 6$ have found that poststroke depression has a negative effect on recovery of functions of daily life activities, but other authors ${ }^{7-9}$ have not.

The reason for this controversy could be the confusing effect of antidepressant drugs, as some physicians tend to use these drugs in patients with poststroke depression, whereas others are reluctant to do so. ${ }^{10}$ Because some authors $^{11}$ have reported a positive effect of some antidepressant drugs on the functional recovery of patients with stroke, it is possible that in treated patients the positive effect of these drugs may have counterbalanced the deleterious influence of the depression.

We think that a randomised study comparing treated and untreated patients with poststroke depression is unethical. However, we have data from a cohort of patients with stroke who were followed up for other research purposes between June 1994 and July 1997. These patients were under the care of physicians, some of whom were inclined to treat poststroke depression and some of whom were not. We decided retrospectively to examine this data set. Our working hypothesis was the following: if poststroke depression has a deleterious influence on rehabilitation, but this effect is counterbalanced by the administration of antidepressant drugs, then an interaction should be found between the results of the rehabilitation process and the presence of a (treated or untreated) poststroke depression. The worst results should be obtained by untreated depressed patients.

Subjects and methods

Subjects were selected from among inpatients consecutively admitted from June 1994 to June 1997 to the Rehabilitation Center Clinica Santa Lucia after a recent stroke. During those years, both the clinical aspects of poststroke depression and the rehabilitation process had been assessed with valid and reliable measures during research programs aiming to investigate: (a) the phenomenological aspects ${ }^{12} 13$ and the anatomical correlates ${ }^{14}$ of poststroke depression, and $(b)$ the variables associated with a positive outcome of rehabilitation. ${ }^{9}$

Furthermore, some patients had been treated and others had not (after the initial assessment of the variables under study) 
Table 1 Characteristics of non-depressed and depressed patients with stroke at the entry and at the end of the study

\begin{tabular}{llll}
\hline & Non-depressed & Depressed but treated & Depressed and non-treated \\
\cline { 2 - 4 } & $(n=15)$ & $(n=24)$ & $(n=25)$ \\
\hline Age (y ) & $63.6(12.1)$ & $60.5(8.9)$ & $62.1(14.7)$ \\
Sex ( M/F) & $9 / 6$ & $14 / 10$ & $13 / 12$ \\
Interval from stroke (months) & $3.1(1.2)$ & $2.9(1.3)$ & $3.2(1.3)$ \\
Years of schooling & $10.1(6.8)$ & $7.5(5.04)$ & $10.2(5.57)$ \\
Side of stroke (left/right) & $8 / 7$ & $10 / 14$ & $9 / 16$ \\
HDRS Scores (entry/end) & $6.7(4.7) / 5.06(3.2)$ & $16.0(6.4) / 6.51(7.9)$ & $18.6(7.7) / 13.2(6.3)$ \\
Canadian NS scores (entry/end) & $7.2(2.3) / 10.4(1.3)$ & $5.7(1.7) / 7.9(2.1)$ & $5.9(2.4) / 7.5(2.8)$ \\
Barthel I scores (entry/end) & $51.3(25.2) / 90.7(14.5)$ & $35.0(18.2) / 74.4(21.5)$ & $38.4(31.3) / 63.2(29.4)$ \\
Rivermead MI scores (entry/end) & $5.4(4.4) / 11.6(3.4)$ & $2.4(2.1) / 8.9(3.6)$ & $3.9(5.0) / 6.8(4.6)$ \\
\hline
\end{tabular}

Values in parentheses are SD.

according to the opinions of their respective physicians. The records of all patients who had undergone a standard assessment of poststroke depression and a rehabilitation programme in that period were taken into account. The patients included in the study met the following criteria: (a) Interval between stroke and admission to the clinic of 1 to 4 months; $(b)$ monohemispheric stroke established by a single CT; (c) no history of strokes and depressive disorders or other important psychiatric disturbances; (d) a decision to treat poststroke depression or not (if a significant level of depression has been detected) based on the general a priori opinion of their physician and not on the severity of depression. Records of 290 patients were examined and 64 patients were included in this study.

\section{ASSESSMENT OF POSTSTROKE DEPRESSION}

The assessment of poststroke depression included a structured psychiatric interview and the administration of the Hamilton depression rating scale (HDRS), which is a well known general depression scale. ${ }^{15}$ Forty nine patients who on the structured psychiatric interview and on the depression rating scale had met the DSM-III-R ${ }^{16}$ diagnostic criteria of (major or minor) depression, were considered as depressed. The remaining 15 patients were classified as non-depressed.

\section{PHARMACOLOGICAL TREATMENT OF POSTSTROKE} DEPRESSION

During the period considered for our research, some physicians of the Clinic Santa Lucia tended to treat poststroke depression with the serotonine reuptake inhibitor fluoxetine or with other antidepressant drugs, whereas other physicians were reluctant to use antidepressant drugs in patients with stroke. Twenty four of the depressed patients with stroke had been treated in our sample, whereas the other 25 had not received antidepressant drugs. Twenty three out of 24 patients received fluoxetine in monotherapy, with dosages ranging between 20 and $40 \mathrm{mg} /$ day and the remaining patient underwent treatment with amitryptiline.

\section{ASSESSMENT OF THE OUTCOME OF THE}

REHABILITATION PROGRAMME

The outcome of rehabilitation was evaluated by computing scores obtained by our three groups of patients with stroke before, during, and at the end of the rehabilitation programme with the following impairment and disability scales: (1) the Barthel index, ${ }^{17}$ the scores of which range between 0 and 100 - the top scores implying complete functional independence in daily life activities; (2) the revised and validated version of the Canadian neurological scale, ${ }^{18}$ the higher scores of which indicate better neurological performances; (3) the Rivermead mobility index, ${ }^{19}$ which assesses the patient's ability in 15 common daily movements with the highest score corresponding to complete motor ability.

The baseline assessment made at the entry into the clinic, the intermediate assessment made after 1 month of rehabilitation, and the final assessment made at the end of the rehabilitation programme were used to evaluate the effect of the main variables (groups and repeated measures) and their interaction with the results obtained with the impairment and disability scales (Canadian neurological scale, Barthel index, and Rivermead motility index). Data were subjected to a two way multivariate analysis of variance (MANOVA) repeated measures analysis. Post hoc comparisons were by Tukey test for unequal sample size when the effect of the main factors or the interaction reached significance.

\section{Results}

The characteristics of the 49 depressed (24 treated and 25 non-treated) and the 15 non-depressed patients with stroke who entered the study are reported in table 1.

Age, sex, education level, hemispheric side of lesion, and interval between the onset of stroke and the beginning of the rehabilitation programme were similar among groups. On the contrary, severity of depression and scores obtained at the entry of the study with the Canadian neurological scale, Barthel index, and Rivermead mobility index were worse in depressed than in non-depressed patients with stroke. On the other hand, neither the severity of baseline depression nor baseline scores obtained with impairment and disability scales were different in treated and untreated depressed patients. The fact that scores obtained on the HDRS were similar in treated and untreated depressed patients with stroke, supports our assumptions that the decision to treat or not was based on the a priori opinion of their physicians and not on the severity of depression.

A one way ANOVA repeated measures comparing the HDRS for the treated and nontreated depressed patients with stroke, showed a better recovery from depression in the treated than in the untreated group $(\mathrm{p}<0.01)$ 

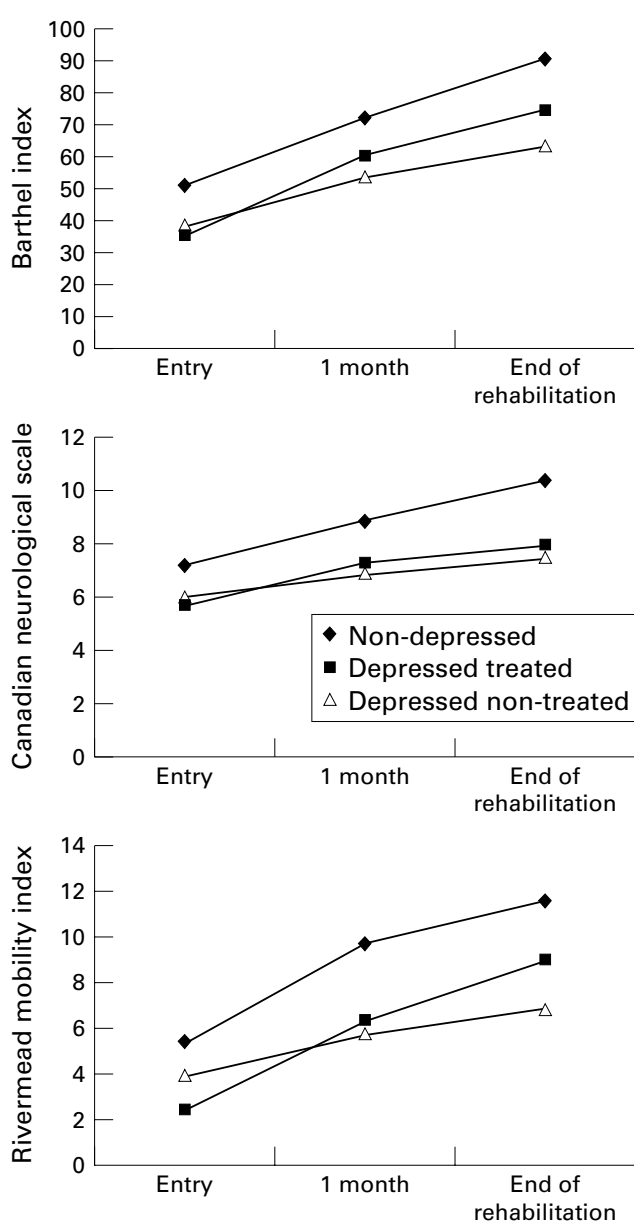

Figure 1 Mean scores on the Barthel index, Canadian neurological scale, and Rivermead mobility index obtained by the three groups of patients at the times studied.

RELATION BETWEEN PRESENCE OF POSTSTROKE DEPRESSION, ADMINISTRATION OF

ANTIDEPRESSANT DRUGS, AND OUTCOME OF THE REHABILITATION

Figure 1 shows the scores obtained with the Canadian neurological scale, Barthel index, and Rivermead mobility index at entry into the study, during, and at the end of the rehabilitation programme by non-depressed and by treated and non-treated depressed patients with stroke. The main scores obtained by the three groups of patients on the same functional scales at the entry and end of the rehabilitation programme, are also shown in table 1.

For all three measures a non-significant difference was found in the group factor; a significant result was obtained with the repeated measure variables and a significant (groups $\times$ repeated measures) interaction was found (Rao's $R(12,112) 3.07$; $<<0.001)$. This interaction was due to the fact that improvement after rehabilitation was higher in nondepressed and in depressed but treated patients with stroke, whereas non-treated depressed patients showed a much lower level of improvement. A specific effect analysis showed that this intergroup difference was particularly significant on the Rivermead mobility index ( $F$ (2, 122) 8.2 ; p $<0.0001)$ and less significant on the Canadian neurological scale $(F(2,122)$
3.7; $\mathrm{p}<0.01)$ and on the Barthel index $(F(2$, 122) $2.8 ; \mathrm{p}<0.03)$.

The post hoc comparison between treated and non-treated depressed patients with stroke showed a significant group $\times$ treatment interaction with the Rivermead mobility index $(p<0.001)$ and Barthel index $(p<0.02)$, but not with the Canadian neurological scale $(\mathrm{p}<0.16)$.

\section{Discussion}

The aim was to evaluate the influence of poststroke depression and antidepressant treatment on functional recovery in patients with stroke undergoing rehabilitation therapy. In particular, we intended to see if the negative effect of poststroke depression on functional recovery could be counterbalanced by the administration of antidepressant drugs. The results, showing that non-treated depressed patients present the lowest rate of functional recovery, whereas depressed but treated patients with stroke have a recovery rate similar to the non-depressed ones, clearly support this hypothesis. This claim is strengthened by two aspects of our study which suggest that the particularly modest results obtained by the untreated depressed patients were due to the harmful influence of psychological factors, and not to uncontrolled sampling bias between treated and non-treated patients. The first refers to the fact that at the beginning of the study, the two groups of depressed patients were indistinguishable in severity of depression and measure of impairment. The second refers to the fact that a different level of interaction between groups and treatment was found on the different outcome measures considered. This interaction was significant on the Rivermead mobility index and on the Barthel index (which are disability scales that examine behavioural aspects and are, therefore, more sensitive to the influence of psychological factors) and non-significant on the Canadian neurological scale (which examines more basic neurological impairment and is, therefore, less sensitive to the effect of these factors). The effects of antidepressant drugs on mood and functional recovery could be, at least partly, independent. Dam et $a l^{11}$ have shown that the noradrenaline (norepinephrine) reuptake blocker maprotiline and the serotonine reuptake inhibitor fluoxetine have a similar effect on mood, but a different one on functional recovery; both improve the HDRS scores, but only fluoxetine actually improves functional recovery, whereas maprotiline hinders it. Future investigation is needed to clarify if the association between functional improvement and reduction of the depressive symptomatology found in our study with fluoxetine is seen with other antidepressant drugs. Clarification of this issue could help establish the most favourable drug for the treatment of poststroke depression.

1 House A. Mood disorders after stroke: a review of the evidence. Int f Geriatr Psychiatry 1987;2:211-21.

2 Starkstein SE, Robinson RG. Affective disorders and cerebral vascular disease. Br F Psychiatry 1989;154:170-82. 3 Andersen G, Vestergaard k, Riis JO, et al. Incidence of postAndersen $\mathrm{G}$, Vestergaard $\mathrm{k}$, Rilis $\mathrm{JO}$, et al. Incidence of post-
stroke depression during the first year in a large unselected 
stroke population determined using a valid standardized rating scale. Acta Psychiatr Scand 1994;90:190-5

Robinson RG, Bolla-Wilson K, Kaplan E, et al. Depression influences intellectual impairment in stroke patients. $\mathrm{Br} \mathcal{F}$ Psychiatry 1986;148:541-7.

5 Parikh RM, Robinson RG, Lypsey JR, et al. The impact of post stroke depression on recovery in activities of daily liv-
ing over a 2-year follow-up. Arch Neurol 1990;47:785-9.

6 Hermann N, Black SE, Lawrence J, et al. The Sunnybrook stroke study. A prospective study of depressive symptoms and functional outcome. Stroke. 1998;29:618-24.

7 Synyor D, Jacques P, Kaloupek DG, et al. Post stroke depression and lesion location. Brain 1986;109:537-46.

8 Loong CK, Kenneth NKC, Paulin ST. Post-stroke depression: outcome following rehabilitation. Aust $N Z \mathcal{F}$ Psychiatry 1995;29:609-14.

9 Paolucci S, Antonucci G, Pratesi L, et al. Poststroke depression and its role in rehabilitation of inpatients. Arch Phys

10 Fann JR, Tucker GJ. Mood disorders with general medical conditions. Curr Opin Psichiatry 1995;8:13-18.

11 Dam H, Pedersen HE, Ahlgren P. Depression among Dam H, Pedersen HE, Ahlgren P. Depression among patients with stroke. Acta Psychiatr Scand 1989;80:118-24. findings concerning a new scale for the assessment of depression and related symptoms in stroke patients. Ital 7 Neurol Sci 1995;16:439-51.

13 Gainotti G, Azzoni A, Razzano C, et al. The post-stroke depression rating scale: a test specifically devised to investigate affective disorders of stroke patients. F Clin Exp Neuropsychol 1987;19:340-56.

14 Gainotti G, Azzoni A, Gasparini F, et al. Relation of lesion location to verbal and non-verbal mood measures in stroke patients. Stroke 1997;28:2145-9.

15 Hamilton H. A rating scale for depression. I Neurol Neurosurg Psychiatry 1969;23:56-62.

16 American Psychiatric Association. Diagnostic and statistical manual of mental disorders (DSM III-R). 3rd ed revised. Washington, DC: APA, 1987.

17 Mahoney F, Barthel DW. Functional evaluation. The Barthel index. Maryland State Medical fournal of Rehabilitation 1965;14:61-5.

18 Coté R, Battista RN, Wolfonson C, et al. The Canadian neurological scale; validation and reliability assessment . Neurology 1989;39:638-43.

19 Collen FM, Wade DT, Robb DT, et al. The Rivermead mobility index: a further development of the Rivermead motor assessment. International Disability Studies 1991;13: $50-4$ 